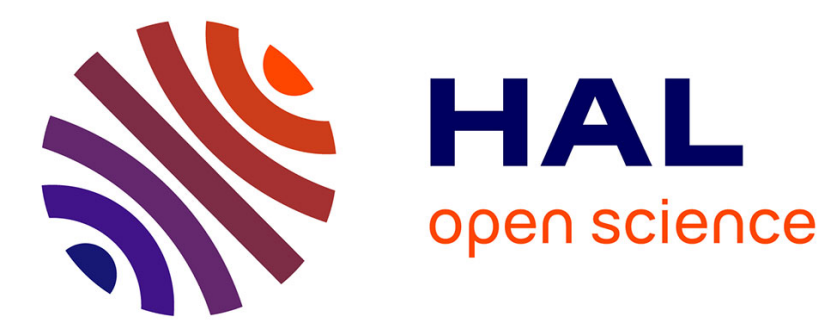

\title{
Nuclear elasticity applied to giant resonances of fast rotating nuclei
}

\author{
S. Jang, A. Bouyssy
}

\section{To cite this version:}

S. Jang, A. Bouyssy. Nuclear elasticity applied to giant resonances of fast rotating nuclei. Physical Review C, 1987, 36, pp.1587-1594. 10.1103/PhysRevC.36.1587 . in2p3-00022702

HAL Id: in2p3-00022702 https://hal.in2p3.fr/in2p3-00022702

Submitted on 20 Nov 2020

HAL is a multi-disciplinary open access archive for the deposit and dissemination of scientific research documents, whether they are published or not. The documents may come from teaching and research institutions in France or abroad, or from public or private research centers.
L'archive ouverte pluridisciplinaire $\mathbf{H A L}$, est destinée au dépôt et à la diffusion de documents scientifiques de niveau recherche, publiés ou non, émanant des établissements d'enseignement et de recherche français ou étrangers, des laboratoires publics ou privés. 


\title{
Nuclear elasticity applied to giant resonances of fast rotating nuclei
}

\author{
S. Jang \\ Institut des Sciences Nucléaires, 38026 Grenoble Cédex, France \\ A. Bouyssy \\ Division de Physique Théorique, Institut de Physique Nucléaire, 91406 Orsay, France
}

\begin{abstract}
Isoscalar giant resonances in fast rotating nuclei are investigated within the framework of nuclear elasticity by solving the equation of motion of elastic nuclear medium in a rotating frame of reference. Both Coriolis and centrifugal forces are taken into account. The nuclear rotation removes completely the azimuthal degeneracy of the giant resonance energies. Realistic large values of the angular velocity, which are still small as compared to the giant resonance frequencies, are briefly reviewed in relation to allowed high angular momenta. It is shown that for the $A=150$ region, the Coriolis force is dominating for small values $(\leqslant 0.05)$ of the ratio of angular velocity to resonance frequency, whereas the centrifugal force plays a prominent part in the shift of the split resonance energies for larger values of the ratio. Typical examples of the resonance energies and their fragmentation due to both rotation and deformation are given.
\end{abstract}

\section{INTRODUCTION}

In a previous paper $^{1}$ (hereafter referred to as I), we investigated the isoscalar giant resonances of deformed nuclei within the framework of the nuclear elasticity. In particular, we turned our attention to the fragmentation of the giant monopole and quadrupole resonances. It was thus shown how the nuclear surface deformation contributes to the fragmentation through the coupling between the monopole and quadrupole modes of oscillation.

In the present paper we extend the concept of nuclear elasticity to rotating nuclei and deal with the isoscalar giant resonances of rotating elastic nuclear matter. The origin of the nuclear elasticity can be found in the fact that the equations of motion formulated in the dynam$\mathrm{ics}^{2,3}$ of nuclear fluid in the framework of the timedependent Hartree-Fock theory can be approximated for some collective motions, such as the giant resonances, by the Lamé equation which governs the classical theory of elasticity. Thus the isoscalar giant resonances of spherical nuclei ${ }^{2,3}$ and those of deformed nuclei ${ }^{1}$ have been fully studied recently in the spirit of the nuclear elastic vibration.

In a recent experiment, Newton et $a l .^{4}$ analyzed the high energy tail of $\gamma$ rays emitted following ${ }^{40} \mathrm{Ar}$ induced reactions and suggested that the nuclear giant dipole resonance may be excited in a nucleus which rotates with very high angular momentum. Regarding the isovector giant dipole resonance of rotating nuclei, there have been several theoretical investigations in the past few years. For example, calculations of the position and splitting of the isovector giant dipole resonance were first performed on the basis of a simple schematic model of a rotating harmonic oscillator. ${ }^{5,6}$ More elaborate models ${ }^{7,8}$ were then applied to the same problem. In a recent paper, ${ }^{9}$ we also studied the isovector giant reso- nances of arbitrary multipolarity in fast rotating nuclei by solving the invicid two-fluid equation of relative motion written in a rotating frame of reference. Though the description we used in Ref. 9 is a rather semiclassical one, the resulting expressions display in a quite simple way general features of isovector giant resonances of fast rotating nuclei. In particular, we have shown how the splitting of the giant resonances arises from rotating deformed nuclei.

For the rotating elastic nuclear matter, we have to transform first the equation of motion in $I$ into one in a rotating frame of reference. Generally, the effective force in the new equation of motion in the rotating coordinates contains the terms corresponding to Coriolis and centrifugal forces. The equation of motion of the rotating nuclear matter is, on the whole, more complicated than the corresponding equation of motion obtained in the two-fluid hydrodynamical model. ${ }^{9}$ The reason is that the elastic medium under the influence of external potentials is not free from stress, even in the initial equilibrium state, and thus the usual expression for the elastic stress in the equation of motion in I has to be slightly modified by adding pressure-dependent terms. Nevertheless, if the density of the elastic nuclear matter is assumed to be constant, the equation of motion can then preserve the same form of the rotational forces as in the case of the two-fluid model, except that the displacement vector now takes part in the force in place of the ordinary radial vector. However, the presence of the rotational forces in the equation of motion prevents us from obtaining an analytical solution of the equation, whatever forms these forces may take.

The derivation of the equation of motion in a rotating frame of reference is described in Sec. II. This is followed by a method of solution of the equation. The perturbation method is shown to be of great utility for the treatment of the equation of motion that contains rota- 
tional forces. Section IV is devoted to discussions of particular examples of the resonance energies in terms of the angular velocity of rotating deformed nuclei. The meaning of nuclear fast rotations is briefly discussed in relation to high angular momenta which can be accomodated in nuclei. A summary and final conclusions are presented in Sec. V.

\section{EQUATION OF MOTION OF ROTATING ELASTIC NUCLEI}

As shown in I, the equation of motion of a uniform perfectly elastic nuclear medium in inertial system is given by

$$
(\lambda+2 \mu) \boldsymbol{\nabla}(\boldsymbol{\nabla} \cdot \mathbf{u})-\mu \boldsymbol{\nabla} \times \boldsymbol{\nabla} \times \mathbf{u}+\mathbf{F}=\rho \frac{\partial^{2} \mathbf{u}}{\partial t^{2}},
$$

where $\mathbf{u}$ is the displacement vector, $\mathbf{F}$ the external body force, and $\rho$ the nuclear density. For the nuclear elasticity the Lamé coefficients $\lambda$ and $\mu$ take the forms ${ }^{1}$

$$
\begin{aligned}
& \lambda=\left(\frac{K}{9}-\frac{2}{15} \frac{\hbar^{2}}{m^{*}} k_{f}^{2}\right) \rho, \\
& \mu=\frac{1}{5} \frac{\hbar^{2}}{m^{*}} k_{f}^{2} \rho,
\end{aligned}
$$

where $K$ is the nuclear compressibility, $m^{*}$ the effective nucleon mass, and $k_{f}$ the Fermi momentum. It is also noticed that the quantity $\lambda+\frac{2}{3} \mu$ is the compression modulus of the elastic medium; that is, the ratio of isotropic pressure to fractional rate of decrease of volume. The Lamé coefficient $\lambda$ is also related to the Landau parameter $F_{0}$ by

$$
\lambda=\frac{1}{5} \frac{\hbar^{2}}{m^{*}} k_{f}^{2} \rho\left(1+\frac{5}{3} F_{0}\right) .
$$

Generally, a dynamical equation of motion expressed in the system of rotating coordinates has the same form as the original equation of motion written in the inertial system, except for the body force term, which now becomes the effective body force. This was the case in the two-fluid model we formulated in a previous paper. ${ }^{9}$ Therefore, to an observer, in the rotating system it appears as if the body is moving under the influence of this effective force, which is represented by

$$
\mathbf{F}_{\text {eff }}=\mathbf{F}-2 \rho\left(\boldsymbol{\Omega} \times \frac{d \mathbf{r}}{d t}\right)-\rho \boldsymbol{\Omega} \times(\boldsymbol{\Omega} \times \mathbf{r}),
$$

where $\boldsymbol{\Omega}$ is the angular velocity. The velocity in the inertial system $\mathbf{v}_{i}$ is related to the velocity in the rotating system $\mathbf{v}$ by $\mathbf{v}_{i}=\mathbf{v}+\boldsymbol{\Omega} \times \mathbf{r}$. The second term in the effective force (2.5) represents the Coriolis force and third term denotes the centrifugal force. If the angular velocity is time dependent, an extra term, $-\rho \mathbf{r} \times d \boldsymbol{\Omega} / d t$, is to be added to the effective force.

The fact that the displacement vector $\mathbf{u}$ plays the role of the usual radial vector $\mathbf{r}$ in the classical dynamics necessitates a careful formulation of the effective force in the approach of nuclear elasticity. Furthermore, the elastic medium under the influence of external potentials is not free from stress, even in the initial equilibrium state, and thus the actual form of the term corresponding to the centrifugal force is slightly more complicated than the term shown in Eq. (2.5). For constant density, however, the effective force can take the same form as before, except that the radial vector is now replaced by the displacement vector. Let the position vector of a typical point $P_{0}$ of the elastic body referred to the origin of a fixed rectangular cartesian system be $\mathbf{s}$. At time $t$, the representative point $P_{0}$ has moved to a point $P$ with position vector $\mathbf{r}$, and the position vector of the point $P$ relative to $P_{0}$ is given by the displacement vector $\mathbf{u}$ with $\mathbf{u}=\mathbf{r}-\mathbf{s}$. In terms of general orthogonal curvilinear coordinates $\left(q_{1}, q_{2}, q_{3}\right)$, the position vectors $\mathbf{s}$ and $\mathbf{r}$ can be written in the forms $\mathbf{s}=\mathbf{s}\left(q_{1}, q_{2}, q_{3}\right), \mathbf{r}=\mathbf{r}\left(q_{1}, q_{2}, q_{3}, t\right)$, and it follows that $\mathbf{u}=\mathbf{u}\left(q_{1}, q_{2}, q_{3}, t\right)$. Therefore, for constant density and in the absence of other potentials such as the Coulomb potential, the linearized equation of motion now takes the form

$$
\begin{aligned}
(\lambda+2 \mu) \boldsymbol{\nabla}(\boldsymbol{\nabla} \cdot \mathbf{u})-\mu \boldsymbol{\nabla} \times \boldsymbol{\nabla} & \times \mathbf{u}-2 \rho \boldsymbol{\Omega} \times \frac{\partial \mathbf{u}}{\partial t} \\
& -\rho \boldsymbol{\Omega} \times(\boldsymbol{\Omega} \times \mathbf{u})=\rho \frac{\partial^{2} \mathbf{u}}{\partial t^{2}} .
\end{aligned}
$$

Equations (2.5) and (2.6) show that the centrifugal force is or order $\Omega^{2}$, whereas the Coriolis force is of order $\Omega$. We now assume that the displacement vector can be separated into spatial and time parts as $\mathbf{u}(\mathbf{r}, t)=\mathbf{u}(\mathbf{r}) \exp (i \omega t)$. Applying the variational principle to Eq. (2.6), as was done in I, we get, after rather tedious transformations,

$$
\begin{aligned}
\omega^{2} \int \rho|\mathbf{u}|^{2} d \tau= & \lambda \int|\boldsymbol{\nabla} \cdot \mathbf{u}|^{2} d \tau+2 \mu \int \sum_{i, j}\left|e_{i j}\right|^{2} d \tau \\
& +\int \rho \mathbf{u}^{*}(2 i \omega \mathbf{\Omega} \times \mathbf{u}) d \tau \\
& +\int \rho \mathbf{u}^{*} \mathbf{\Omega} \times(\mathbf{\Omega} \times \mathbf{u}) d \tau
\end{aligned}
$$

This is the variational form of the equation of motion which consists of the main equation in the present investigation. Because of the presence of the additional force terms which represent nuclear rotation, we cannot expect to express the frequency $\omega$ in the variational form as in Eq. (3.4) of I. We shall show, however, how the perturbation method enables us to acquire a quite simple expression for the frequency of vibration of rotating nuclei.

Before going closely into the method of solution of Eq. (2.7), we shall now consider briefly the case of an elastic body whose density is a function of $r$ and which, accordingly, is assumed to be in a state of hydrostatic stress before being disturbed by external forces. In a slightly deformed elastic state, the stress is then the sum of the usual stress due to the small displacement $\mathbf{u}$ and the initial hydrostatic stress. The former is given by Eq. (2.2) of $I$ and the latter is expressed as the negative of the original hydrostatic pressure at the initial representative 
points $P_{0}$. Thus the initial stress is shown to be

$$
-p(\mathbf{r}+(-\mathbf{u}))=-[p(\mathbf{r})-\mathbf{u} \cdot \nabla p],
$$

where $p$ is the hydrostatic pressure. Moreover, owing to disturbance due to the small displacement, the density in the disturbed state may be written as $\rho^{\prime}=\rho+\delta \rho$, where $\delta \rho$ is the change in density, which is calculated from the principle of mass conservation.

Assuming further that the body force can be associated with a potential $\Psi$, we can equate the body force $F$ to the gradient of the potential, multiplied by the disturbed density. Since the nucleus under consideration is rotating, the potential $\Psi$ may comprise the centrifugal term $\psi$,

$$
\psi=\frac{1}{2}\left[(\Omega r)^{2}-(\boldsymbol{\Omega} \cdot \mathbf{r})^{2}\right],
$$

as well as the Coulomb potential. It is to be remarked that the use of the potential $\Psi$ instead of the explicit form of the centrifugal force modifies the expression (2.5) so that the centrifugal term is now included in $\mathbf{F}$.

When we introduce the initial stress as well as the body-force term into the equation of motion written in terms of the stress-strain tensors, such as Eq. (3.1) of I, we see that the equation of motion now contains the terms

$$
-\nabla(p-\mathbf{u} \cdot \nabla p)+\rho \nabla \Psi+\delta \rho \nabla \Psi .
$$

When we neglect the contribution arising from the change in density and omit the initial stress, the expression (2.9) reduces then to the term which represents the usual centrifugal force. The pressure in (2.9) may also be associated with the potential $\Psi$ by assuming that the pressure force in the hydrostatic equilibrium state, which is the negative of the pressure gradient, counterbalances the body force. Thus, for the rotating body with angular velocity $\Omega$, we have ${ }^{10}$

$$
\nabla p=\rho \nabla \Psi \text {. }
$$

The pressure force will, in general, be very small, unless there is a very large pressure gradient. When we keep only the centrifugal term $\psi$ in $\Psi$, we see, in view of the relation (2.10), that all terms of (2.9) are of order $\Omega^{2}$. If there is no rotation, the potential $\Psi$ reduces practically to the Coulomb term and the contribution from the initial stress to the usual nuclear elastic vibration will be inconsiderable, unless the relation $(2.10)$ is reviewed so as to be still significant with regard to other specific external potentials. The form (2.9) of the centrifugal effect for the rotating elastic body contrasts with the corresponding term in the equation of motion (2.6) for constant density. We shall come back to the expression (2.9) on the occasion of practical evaluation of various rotational effects.

\section{PERTURBATION TREATMENT OF ROTATIONAL EFFECTS}

Though an analytical solution of Eq. (2.6) cannot be expected, the equation of motion of this type can well be treated using the perturbation method, provided the magnitude of angular velocity is much smaller than the unperturbed initial frequency of oscillation without rotation. From the quantum mechanical point of view the nuclear rotation makes sense only for deformed nuclei, whereas the rotation of a spherical body has meanings in the classical picture. In the following we first concentrate on the rotational effects by solving Eq. (2.6) without including the degree of freedom of deformation in the formulation and we subsequently take into account the nuclear surface deformation.

The displacement vector $\mathbf{u}$ as well as the frequency $\omega$ are now expanded in powers of $\Omega / \omega_{0}$ as

$$
\begin{aligned}
& \mathbf{u}=\mathbf{u}_{0}+\left(\frac{\Omega}{\omega_{0}}\right) \mathbf{u}_{1}+\left(\frac{\Omega}{\omega_{0}}\right)^{2} \mathbf{u}_{2}+\cdots, \\
& \omega=\omega_{0}+\left(\frac{\Omega}{\omega_{0}}\right) \omega_{1}+\left(\frac{\Omega}{\omega_{0}}\right)^{2} \omega_{2}+\cdots,
\end{aligned}
$$

where $\omega_{0}$ is the unperturbed initial frequency of oscillation without rotation. Upon introducing the expansion (3.1) and (3.2) into Eq. (2.6) and collecting the terms of the same power of $\Omega / \omega_{0}$ up to second order, we get

$$
\begin{aligned}
& (\lambda+2 \mu) \boldsymbol{\nabla}\left(\boldsymbol{\nabla} \cdot \mathbf{u}_{0}\right)-\mu \boldsymbol{\nabla} \times \boldsymbol{\nabla} \times \mathbf{u}_{0}=-\omega_{0}^{2} \rho \mathbf{u}_{0}, \\
& (\lambda+2 \mu) \boldsymbol{\nabla}\left(\boldsymbol{\nabla} \cdot \mathbf{u}_{1}\right)-\mu \boldsymbol{\nabla} \times \boldsymbol{\nabla} \times \mathbf{u}_{1}-2 i \frac{\omega_{0}^{2}}{\Omega} \rho \mathbf{\Omega} \times \mathbf{u}_{0}=-\rho\left(\omega_{0}^{2} \mathbf{u}_{1}+2 \omega_{0} \omega_{1} \mathbf{u}_{0}\right), \\
& (\lambda+2 \mu) \boldsymbol{\nabla}\left(\boldsymbol{\nabla} \cdot \mathbf{u}_{2}\right)-\mu \boldsymbol{\nabla} \times \boldsymbol{\nabla} \times \mathbf{u}_{2}-2 i \frac{\omega_{0}^{2}}{\Omega} \rho \mathbf{\Omega} \times \mathbf{u}_{1}-2 i \frac{\omega_{0} \omega_{1}}{\Omega} \rho \mathbf{\Omega} \times \mathbf{u}_{0}-\left(\frac{\omega_{0}}{\Omega}\right)^{2} \boldsymbol{\Omega} \times\left(\boldsymbol{\Omega} \times \mathbf{u}_{0}\right) \\
& =-\rho\left(\omega_{0}^{2} \mathbf{u}_{2}+2 \omega_{0} \omega_{1} \mathbf{u}_{1}+\omega_{1}^{2} \mathbf{u}_{0}+2 \omega_{0} \omega_{2} \mathbf{u}_{0}\right) .
\end{aligned}
$$

Multiplying Eq. (3.3) by $\mathbf{u}_{0}^{*}$ and integrating the result by parts, we obtain

$$
\begin{aligned}
\omega_{0}^{2} \int \rho\left|\mathbf{u}_{0}\right|^{2} d \tau= & \lambda \int\left|\boldsymbol{\nabla} \cdot \mathbf{u}_{0}\right|^{2} d \tau \\
& +2 \mu \int \sum_{i, j}^{3}\left|e_{i j}^{(0)}\right|^{2} d \tau,
\end{aligned}
$$

where $e_{i j}$ are the strain tensors which were defined in I. In obtaining Eq. (3.6), we have taken into account the boundary condition which states that the strain-stress tensor components vanish at the nuclear surface. Equation (3.6) is nothing but the variational expression we have already derived for the original Lamé equation 
which governs the motion of a perfect elastic medium. The explicit form of the function $\mathbf{u}_{0}$ has been shown ${ }^{1}$ to be

$$
\mathbf{u}_{0}=u_{0 r} \widehat{\mathbf{n}}_{r}+u_{0 \theta} \widehat{\mathbf{n}}_{\theta}+u_{0 \phi} \widehat{\mathbf{n}}_{\phi},
$$

where

$$
\begin{aligned}
& u_{0 r}=U_{l}(r) Y_{l m}(\theta, \phi), \\
& u_{0 \theta}=V_{l}(r) \frac{\partial Y_{l m}}{\partial \theta}, \\
& u_{0 \phi}=V_{l}(r) \frac{1}{\sin \theta} \frac{\partial Y_{l m}}{\partial \phi},
\end{aligned}
$$

and $\widehat{\mathbf{n}}_{r}, \widehat{\mathbf{n}}_{\theta}$, and $\hat{\mathbf{n}}_{\phi}$ denote, respectively, unit vectors in the directions of $r, \theta$, and $\phi$. In Eq. (3.8), $Y_{l m}(\theta, \phi)$ are the spherical harmonics and the radial functions $U_{l}(r)$ and $V_{l}(r)$ for constant density are expressed in terms of spherical Bessel functions $j_{l}(x)$ as

$$
\begin{aligned}
& U_{l}(r)=A_{l} \frac{1}{h^{2}} \frac{\partial}{\partial r} j_{l}(h r)+C_{l} \frac{1}{k^{2}} \frac{l(l+1)}{r} j_{l}(k r), \\
& V_{l}(r)=A_{l} \frac{1}{h^{2}} \frac{1}{r} j_{l}(h r)+C_{l} \frac{1}{k^{2}} \frac{1}{r} \frac{\partial}{\partial r}\left[r j_{l}(k r)\right],
\end{aligned}
$$

where

$$
h^{2}=\rho \omega_{0}^{2} /(\lambda+2 \mu) \text { and } k^{2}=\rho \omega_{0}^{2} / \mu .
$$

The constant multipliers $A_{l}$ and $C_{l}$ are determined from the boundary conditions. To be more precise, the boundary condition we have previously stated leads to two expressions for the ratio $C_{l} / A_{l}$. For example,

$$
\frac{C_{l}}{A_{l}}=\frac{2 \frac{d}{d \xi}\left(\frac{j_{l}(\xi)}{\xi}\right)}{\frac{d^{2}}{d \eta^{2}} j_{l}(\eta)+\frac{l(l+1)-2}{\eta^{2}} j_{l}(\eta)},
$$

where $\xi=h R_{0}$ and $\eta=k R_{0}, R_{0}$ being the radius of a spherical nucleus. The other expression for the same ratio was shown in I. Having obtained the eigenvalues $\xi$ and $\eta$, we can proceed to the evaluation of various integrals in which the radial functions $U_{l}$ and $V_{l}$ take part.

Equations (3.4) and (3.5) can now be solved with the help of the standard stationary perturbation technique. To this end, we multiply Eq. (3.4) by $\mathbf{u}_{0}^{*}$ and Eq. (3.3) by $\mathbf{u}_{1}^{*}$ and then integrate the difference between two resulting equations. We then get

$$
\omega_{1}=i \frac{\omega_{0}}{\Omega} \frac{\int \rho \mathbf{u}_{0}^{*}\left(\boldsymbol{\Omega} \times \mathbf{u}_{0}\right) d \tau}{\int \rho\left|\mathbf{u}_{0}\right|^{2} d \tau}
$$

The expression (3.12) represents the rotational effect arising from the Coriolis force alone. We now assume, without losing generality, that the angular velocity $\mathbf{\Omega}$ is directed to the polar axis $\mathrm{Oz}$ of rectangular Cartesian axes $O x y z$ with the center of nucleus as the origin. In spherical polar coordinates, $\mathbf{\Omega}$ then takes the form

$$
\boldsymbol{\Omega}=(\boldsymbol{\Omega} \cos \theta) \widehat{\mathbf{n}}_{r}+(-\boldsymbol{\Omega} \sin \theta) \widehat{\mathbf{n}}_{\theta}+0 \widehat{\mathbf{n}}_{\phi} .
$$

Upon introducing the expression (3.13) into Eq. (3.12), we get, after performing angular integrations,

$$
\omega_{1}=m \omega_{0} \frac{I_{n}}{I_{d}},
$$

where

$$
\begin{aligned}
& I_{\mathrm{n}}=\int \rho\left[2 U_{l}(r) V_{l}(r)+V_{l}^{2}(r)\right] r^{2} d r, \\
& I_{\mathrm{d}}=\int \rho\left[U_{l}^{2}(r)+l(l+1) V_{l}^{2}(r)\right] r^{2} d r .
\end{aligned}
$$

We see that, apart from two radial integrals, the effect of rotation due to the Coriolis force is simply proportional to the azimuthal component $m$ multiplied by the initial unperturbed frequency. The present result is to be compared with the corresponding formula obtained in the two-fluid model. ${ }^{9}$ Since the eigenvalue equation obtained in the framework of the nuclear elasticity is much more complicated than the similar equation used in the hydrodynamical model, ${ }^{9}$ further simplication of the expression (3.14) as a function of eigenvalues cannot be expected even for constant density. The formula (3.14) is unfamiliar to nuclear physicists but not to geophysicists. It is worth mentioning that the classical theory of elasticity has already been applied to the rotational problem of the Earth. ${ }^{10}$ The assumption of constant density allows us to integrate analytically two radial integrals $(3.15 \mathrm{a})$ and (3.15b) (see the Appendix). As we shall see, these are the only integrals to be computed through the present work. The result (3.14) shows that there is no rotational effect for $m=0$ as far as the first order calculation is concerned. The second order formulation removes, however, this restriction.

In a way analogous to the derivation of the first order effect, we can derive the second order frequency of oscillation, $\omega_{2}$. Thus, we get, from Eq. (3.5),

$\left.\omega_{2}=\frac{1}{2 \omega_{0}} \mid \omega_{1}^{2}+\frac{\omega_{0}^{2}}{\Omega^{2}} \frac{\int \rho \mathbf{u}_{0}^{*} \cdot \mathbf{\Omega} \times\left(\mathbf{\Omega} \times \mathbf{u}_{0}\right) d \tau}{\int \rho\left|\mathbf{u}_{0}\right|^{2} d \tau}\right)+\boldsymbol{\epsilon}$,

where

$$
\epsilon=\left(\omega_{1}^{\prime}-\omega_{1}\right) \frac{\int \rho \mathbf{u}_{0}^{*} \mathbf{u}_{1} d \tau}{\int \rho\left|\mathbf{u}_{0}\right|^{2} d \tau},
$$

with

$$
\omega_{1}^{\prime}=\frac{i \omega_{0}}{\Omega} \frac{\int \rho \mathbf{u}_{0}^{*} \cdot\left(\boldsymbol{\Omega} \times \mathbf{u}_{1}\right) d \tau}{\int \rho \mathbf{u}_{0}^{*} \mathbf{u}_{1} d \tau} .
$$

The first and last terms on the right-hand side of Eq. (3.16) clearly represent the second order contribution from the Coriolis force, while the second term shows the centrifugal part. The expression for $\omega_{1}^{\prime}$ is formally the same as for $\omega_{1}$, except that $\mathbf{u}_{1}$ is used in place of $\mathbf{u}_{0}$. Since $\epsilon$ describes the difference between $\omega_{1}^{\prime}$ and $\omega_{1}$, we 
can expect its contribution to $\omega_{2}$ to be small. Actually, this is verified by computing explicitly the first order displacement vector $\mathbf{u}_{1}$ by means of the usual perturbation method; that is, by expanding $\mathbf{u}_{1}$ in terms of $\mathbf{u}_{0}$ as $\mathbf{u}_{1}=\sum_{v} a^{(v)} \mathbf{u}_{0}^{(v)}$

The angular integrals in Eq. (3.16) can easily be evaluated and, finally, $\omega_{2}$ can be expressed in terms of known radial integrals $I_{\mathrm{n}}$ and $I_{\mathrm{d}}$ of Eqs. (3.15). We have

$$
\omega_{2}=\frac{1}{2} \omega_{0}\left\{\left[m \frac{I_{\mathrm{n}}}{I_{\mathrm{d}}}\right]^{2}-\frac{1}{(2 l-1)(2 l+3)}\left[2\left(l^{2}+l+m^{2}-1\right)-\left(l^{2}+l-3 m^{2}\right) \frac{\frac{I}{I_{\mathrm{d}}}}{I^{2}}\right]\right\}+\epsilon .
$$

The result (3.18) is quite simple and its numerical computation is elementary, as in the case of the first order frequency. We see immediately that the second order frequency does not vanish even for $m=0$ and the second order rotational correction does also act to shift the eigenfrequency of $m=0$ mode.

When the density is a function of $r$ and when we keep only the term $\psi$ in the potential $\Psi$, the centrifugal contribution can be evaluated with the help of the expressions of (2.9) and (2.10). When we apply the principle of mass conservation to the total density, the small deviation of density $\delta \rho$ may be equated to $-\nabla \cdot(\rho \mathbf{u})$. Neglecting small quantities of the higher order, the second order frequency is now shown to be

$$
\omega_{2}=\frac{1}{2 \omega_{0}}\left\{\omega_{1}^{2}+\frac{\omega_{0}^{2}}{\Omega^{2}}\left[\int \mathbf{u}_{0}^{*} \cdot \nabla \cdot\left(\rho \mathbf{u}_{0}\right) \cdot \nabla \psi d \tau-\int \mathbf{u}_{0}^{*} \cdot \nabla\left(\rho \mathbf{u}_{0} \cdot \nabla \psi\right) d \tau\right] / I_{\mathrm{d}}\right\}+\epsilon .
$$

The angular integrals involved in this representation are rather cumbersome, but they are still analytically integrable. Thus, the second and third terms in the square brackets on the right-hand side become

$$
-\frac{\Omega^{2}}{(2 l-1)(2 l+3)}\left\{2\left[l(l+1)+m^{2}-1\right] \int \Delta_{l}(r) U_{l}(r) r^{2} d r+\left[3 m^{2}-l(l+1)\right] \int \Delta_{l}(r) V_{l}(r) r^{2} d r\right\} / I_{\mathrm{d}},
$$

where

$$
\Delta_{l}(r)=2 \rho(r)\left[l(l+1) V_{l}(r)-2 U_{l}(r)-r \frac{\partial}{\partial r} U_{l}(r)\right]-U_{l}(r) r \frac{\partial}{\partial r} \rho(r) .
$$

The expression (3.20) contrasts with the corresponding form (3.18), obtained from the density-independent centrifugal potential. In fact, the eigenvalues $h R_{0}$ and $k R_{0}$ that are involved in the integrals of (3.20) are not those evaluated from the eigenvalue equation formulated in $I$. Since both displacement vector and density in the original Lamé equation are now variables, the zero-order equation (3.6) can be solved only numerically and thus the radial functions $U_{l}(r)$ and $V_{l}(r)$ are no longer given by the relations (3.9) and (3.10). Furthermore, according to the formulas (2.2) and (2.3), the Lamé coefficients $\lambda$ and $\mu$ also change their values when the density varies (see Ref. 11). Though the study of the density- dependent nuclear elasticity constitutes an interesting new subject, the complexity of numerical calculations in the present problem may prevent one from gaining physical insight into the problem of rotating nuclear vibration. In the following we therefore turn our attention to the expression (3.16) together with (3.12).

\section{TYPICAL NUMERICAL RESULTS}

When we substitute the results (3.14) and (3.18) into the formula (3.2) for the total frequency of vibration, we get the desired expression to second order for rotating nuclei. Thus,

$\left.\frac{\omega}{\omega_{0}}=1+\left[\frac{\Omega}{\omega_{0}}\right]\left[m \frac{I_{\mathrm{n}}}{I_{\mathrm{d}}}\right]+\left(\frac{\Omega}{\omega_{0}}\right]^{2}\left[\left\{m \frac{I_{\mathrm{n}}}{I_{\mathrm{d}}}\right]^{2}-\frac{1}{(2 l-1)(2 l+3)}\left\{l^{2}+l+m^{2}-1\right)+\frac{1}{2}\left[3 m^{2}-l(l+1)\right] \frac{I_{\mathrm{n}}}{I_{\mathrm{d}}}\right\}+\frac{\epsilon}{\omega_{0}}\right]$.

Upon introducing explicit results of the radial integrals $I_{\mathrm{n}}$ and $I_{\mathrm{d}}$, the ratio $\omega / \omega_{0}$ for a given value of multipolarity $l$ and its projection $m$ can be expressed as a function of $\Omega / \omega_{0}$, which is a measure of the angular velocity of the rotation.

Generally, rotational states with angular momentum $I$ in nuclei can be characterized by their excitation energies $E_{I}$, which usually lie low compared to the singleparticle excitations and which obey the $I(I+1)$ rule.
However, the well-known expression for rotational excitation energies,

$$
E_{I}=A I(I+1)+B(I(I+1))^{2}+\cdots,
$$

converges poorly for $I \gtrsim 10$ (Refs. 12 and 13). A much wider region of convergence can be realized if the angular velocity of nuclear rotation, $\Omega$, is used as an expansion parameter. With the assumption of a rigid rotor, 
the nuclear angular velocity can be expressed ${ }^{13,14}$ as $\Omega=\hbar \sqrt{I(I+1)} / \mathcal{I}$, which relates the angular velocity $\Omega$, the angular momentum $I$, and the nuclear moment of inertia $\mathcal{7}$. It is thus seen that the angular velocity could attain, in principle very large values for higher angular momenta, provided the nuclear moment of inertia remains constant. However, the assumption of constant moment of inertia is not always reliable, as discussed in Ref. 13. The typical values of angular moment which can be accommodated in nuclei have already been established in Ref. 14. In a previous paper ${ }^{9}$, we have also given some examples of angular velocity characterizing various nuclei at different angular momenta. According to these results, reasonable values of angular velocity of rotation are rather small as compared to the giant resonance frequencies. For sufficiently high spin states, e.g., $I=78$ for the ${ }^{154} \mathrm{Sm}$ nucleus and $I=20$ for the ${ }^{24} \mathrm{Mg} \mathrm{nu}-$ cleus, the angular velocity $\Omega$ takes the value of 1.07 $\mathrm{MeV}$ for ${ }^{154} \mathrm{Sm}$ and and $6.59 \mathrm{MeV}$ for ${ }^{24} \mathrm{Mg}$. These values imply that the ratio of the angular velocity to the isoscalar giant quadrupole resonance, $\Omega / \omega_{0}$, amounts to 0.09 and 0.3 , respectively. The large value of the ratio for the ${ }^{24} \mathrm{Mg}$ nucleus is, of course, due to the rather high angular velocities of nuclear rotation which may occur in light nuclei. ${ }^{15}$

Although the ratio $\Omega / \omega_{0}$ in the present values covers a very wide region, it is to be understood that the most realistic large value of the ratio for the $A=150$ region lies somewhere near 0.08 . However, when we deal with light nuclei, such as ${ }^{24} \mathrm{Mg}$, a wider region of the ratio, up to 0.3 or more, should be taken into consideration. The ratio $\Omega / \omega_{h}, \omega_{h}$ being the harmonic oscillator frequency, has also been used elsewhere, ${ }^{6,7,16,17}$ instead of the present ratio $\Omega / \omega_{0}$, and some speculative values of the ratio have been tentatively interpolated.

Figure 1 displays the ratio $\omega / \omega_{0}$ for the multipolarities $l=1$ and 2 for nuclei of $A=150$ without deformation $(\delta=0)$. Obviously, the nuclear rotation does not make much sense for spherical nuclei, and thus Fig. 1 is drawn with the intention of showing how the rotation splits the resonance frequencies following values of the azimuthal component and the angular velocity. In this figure, $a\left(a^{\prime}\right), b\left(b^{\prime}\right)$, and $c\left(c^{\prime}\right)$ for $l=1$ stand for $m=0,1$, and -1 , respectively, and $a\left(a^{\prime}\right), b\left(b^{\prime}\right), c\left(c^{\prime}\right), d$ $\left(d^{\prime}\right)$, and $e\left(e^{\prime}\right)$ for $l=2$ denote the components of $m=0$, $1,-1,2$, and -2 , respectively. The thin curves represent the effects of the Coriolis force alone, whereas the broad curves show the effects of both the Coriolis and centrifugal forces. It is seen that for small values of the ratio of angular velocity to resonance frequencythat is, $\Omega / \omega_{0} \leqslant 0.05$ - the effect of the Coriolis force dominates, but the centrifugal force becomes prominent for larger values of the ratio. However, as was already discussed, the range of values of the ratio which is larger than 0.1 is a pure theoretical postulate, at least for nuclei of $A=150$. In Fig. 1 the ordinate value of $\omega / \omega_{0}=1$ corresponds to the unperturbed resonance energies 74.1 $A^{-1 / 3} \mathrm{MeV}$ for $l=1$ and $66.1 A^{-1 / 3} \mathrm{MeV}$ for $l=2$.

For deformed nuclei, $\omega_{0}$ is not a single-valued quantity, but is already split into fragments due to the deformation, as seen in I. A straightforward but hardly feasi-

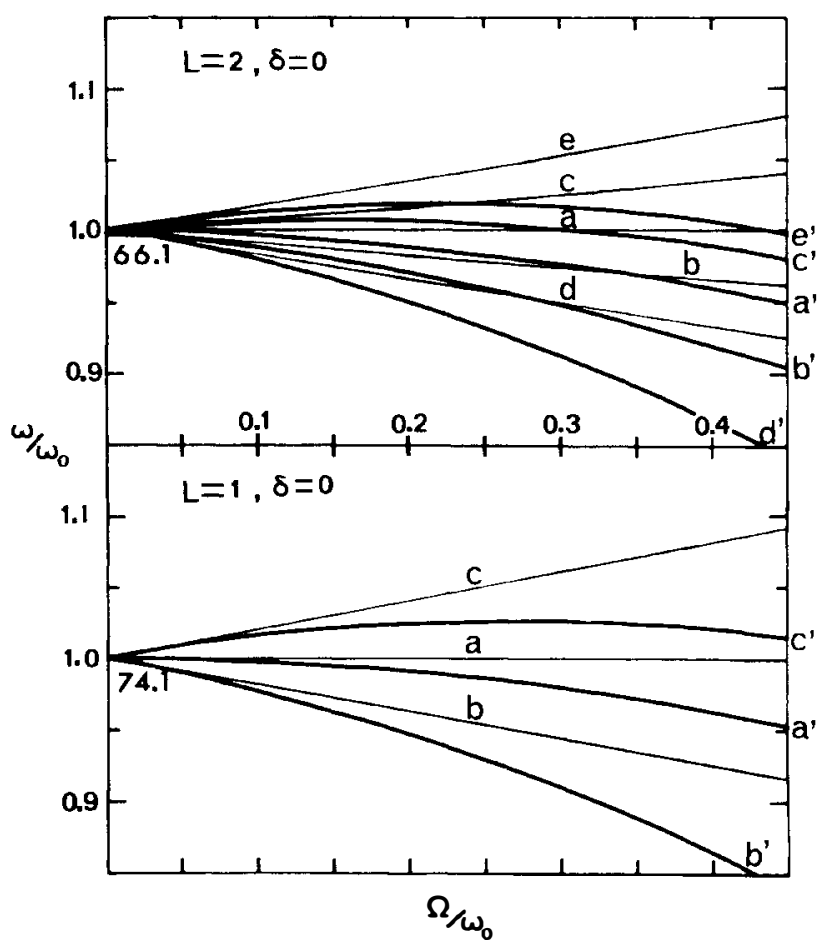

FIG. 1. Rotational multiplets of the isoscalar giant quadrupole and dipole resonances for nuclei of $A=150$ with $\delta=0$. Both angular velocity $\Omega$ and resonance frequency $\omega$ are given in units of $\omega_{0}$, unperturbed frequency without rotation. See the text for further details.

ble method for dealing with the vibration of rotating deformed nuclei is to solve Eq. (2.7) directly in spheroidal coordinates, but the task is laborious and time consuming. Instead, we employ the variational procedure we already used in Ref. 9 in relation with the two-fluid model. The essential point of this method consists of using the deformed radius $R=R_{0}\left(1+\sum \alpha_{2 v} Y_{2 v}(\theta, \phi)\right)$ as the upper limit of all integrals involved in the variational equation. The splitting of the giant resonance energies due to the nuclear surface deformation was thus shown ${ }^{1}$ to be

$$
\omega_{0 m}^{2} \simeq \omega_{0}^{2}\left[1-\left(\frac{b_{l}}{I_{\mathrm{d}}}-\frac{d_{l}}{c_{l}}\right) \zeta_{l, \pm m}\right],
$$

where

$$
\begin{aligned}
\xi_{l, \pm m}=\frac{1}{(2 l-1)(2 l+3)}\left\{\left[l(l+1)-3 m^{2}\right] \epsilon_{3}\right. \\
\left.+\frac{1}{2} l(l+1)\left(\epsilon_{3}+2 \epsilon_{1(2)}\right) \delta_{m 1}\right\}
\end{aligned}
$$

with $\epsilon_{\kappa}=\sqrt{5 / 4 \pi} \beta \cos \left(\gamma-\frac{2}{3} i \pi\right), \beta$ and $\gamma$ being the deformation parameters. The quantities $b_{l}, c_{l}$, and $d_{l}$ are defined in $I$. When we use the same procedure as used for deriving the expression (4.2) in the evaluation of $\omega_{1}$, we now obtain 


$$
\omega_{1}^{\mathrm{def}}=m \omega_{0}^{\prime}\left(I_{\mathrm{n}}+I_{\mathrm{n}}^{\prime}\right) /\left(I_{\mathrm{d}}+I_{\mathrm{d}}^{\prime}\right),
$$

where $I_{\mathrm{n}}^{\prime}$ and $I_{\mathrm{d}}^{\prime}$ are the quantities arising from the nuclear surface deformation. Furthermore, the frequency $\omega_{0}^{\prime}$ on the right-hand side of $\omega_{1}^{\text {def }}$ should represent the frequency of deformed nuclei without rotation; that is, $\omega_{0 m}$ of the expression (4.2). In this case, however, the terms like $\zeta_{l} I_{\mathrm{d}}^{\prime} / I_{\mathrm{d}}$ and $\zeta_{l} I_{\mathrm{n}}^{\prime} / I_{\mathrm{n}}$ are of the order $\epsilon_{\kappa}^{2}$ and thus we can neglect them as far as the first order of the deformation parameter $\epsilon_{\kappa}$ is concerned. The expression $\omega_{1}^{\text {def }}$ we have thus derived now embodies the nuclear surface deformation up to the first order of $\epsilon_{\kappa}$. Similarly, we can reformulate the expression (3.18) so as to become a function of deformation parameter $\epsilon_{\kappa}$. It follows from this argument that a simple way of incorporating the nuclear deformation in the vibration of rotating nuclei is to replace $\omega_{0}$ in Eqs. (3.14) and (3.18) by $\omega_{0 m}$.

As for typical numerical results, we have chosen nuclei of $A=150$ which are known to be well deformed. Moreover, the structural properties of fast rotating nuclei in this region have recently been the subject of many experimental and theoretical studies. ${ }^{13}$ We take a value of 0.2 for the deformation parameter $\delta=\frac{3}{2} \sqrt{5 / 4 \pi} \beta$. Figure 2 displays how the isoscalar giant dipole resonance of rotating deformed nuclei split into different fragments. The energies $70.2 A^{-1 / 3}$ and $82.0 A^{-1 / 3} \mathrm{MeV}$ show the splitting of the resonance energy of prolate nuclei without rotation, whereas $66.3 A^{-1 / 3}$ and $78.0 A^{-1 / 3}$ $\mathrm{MeV}$ correspond to that of oblate nuclei without rotation. The ordinate value 1 corresponds to the resonance energy of spherical nuclei, which is $74.1 A^{-1 / 3} \mathrm{MeV}$. The indices 1, 2, and 3 attached to $p$ (prolate) or $o$ (oblate) stand for the fragments with $m=1,-1$, and 0 , respectively. Figure 2 contrasts much with the corresponding figure for the isovector giant dipole resonance, shown in Ref. 9. Figure 2 shows that the shift of reso-

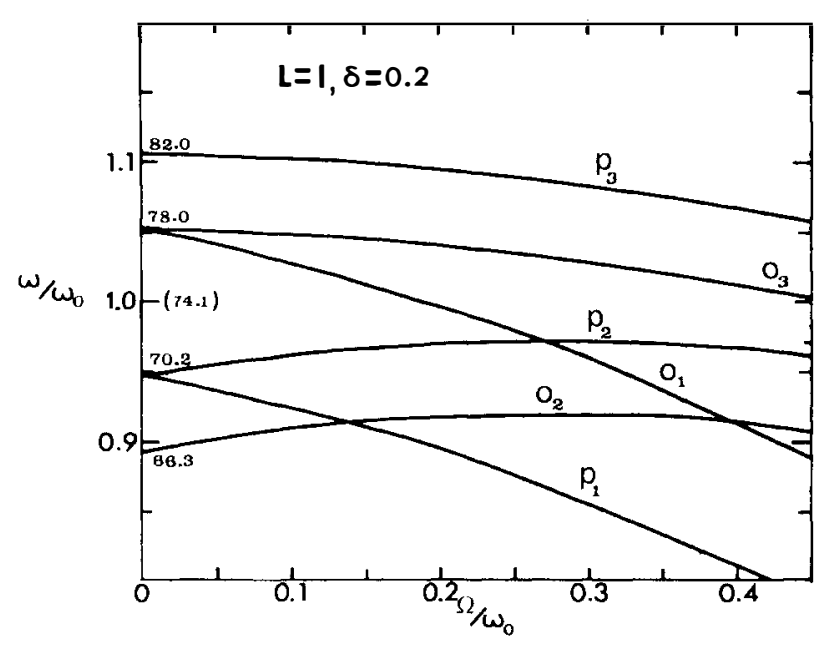

FIG. 2. Fragmentation of the isoscalar giant dipole resonance of rotating prolate and oblate nuclei of $A=150$ with $\delta=0.2$. The resonance frequency $\omega$ is plotted as a function of angular velocity $\Omega$. The unit for both $\omega$ and $\Omega$ is as for Fig. 1 . See the text for further details. nance energies by rotation is very acute only for the fragments corresponding to $p_{1}$ and $o_{1}$, while the other components change little and remain almost the same even for very large values of the ratio $\Omega / \omega_{0}$.

Figure 3 shows the result of the same calculation for the isoscalar giant quadrupole resonance. The ordinate value 1 in this figure represents the giant quadrupole resonance of spherical nuclei, which is $66.1 A^{-1 / 3} \mathrm{MeV}$ in the model of nuclear elasticity. The energies $60.6 A^{-1 / 3}$, 63.3 $A^{-1 / 3}, 68.8 A^{-1 / 3}$, and $71.5 A^{-1 / 3} \mathrm{MeV}$ signify the components of the resonance energies without rotation for either prolate or oblate deformations. The bold curves are for prolate and dashed curves are for oblate. The letters $a, b, c, d$ and $e$ represent the different fragments corresponding to $m=2,-2,1,-1$, and 0 . The shift of the initial resonance energies by rotation is quite pronounced for the curves $a$ and $c$, but not for the other fragments. It is interesting to note that there is essentially no difference between the fragments $b$ and $d$ in oblate nuclei and that for very fast rotation - that is, $\Omega / \omega \simeq 0.4$, which is fairly far from realistic values - the number of fragments is again reduced to 3 , which is the initial multiplet of the resonance energy without rotation. The giant quadrupole resonance in rotating nuclei has also been discussed ${ }^{17}$ by separating the quadrupole oscillation into toroidal, pulsation, and transversely skewed modes in the framework of distorted Fermisurface model. As we observe in the schematic representation of splitting of Fig. 1, the split energies of the isoscalar giant resonances in rotating nuclei are not generally symmetric with respect to the middle line corresponding to the resonance energy of spherical nuclei without rotation, contrary to the case of isovector giant resonance splittings (Refs. 5, 8, and 9). It is interesting to ascertain that a recent calculation ${ }^{18}$ based on the linear response theory reveals the same features.

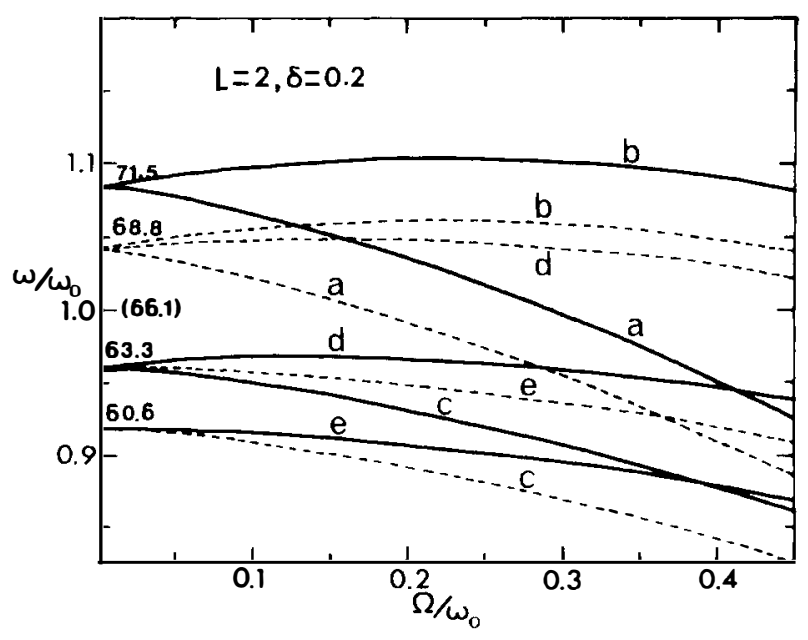

FIG. 3. Fragmentation of the isoscalar giant quadrupole resonance of rotating prolate and oblate nuclei of $A=150$ with $\delta=0.2$. The unit for both $\omega$ and $\Omega$ is as for Fig. 1 . 


\section{SUMMARY AND CONCLUSION}

The main purpose of the present work was to study the giant resonances of rotating nuclei, especially the isoscalar type of giant dipole and quadrupole resonances, by extending the concept of nuclear elasticity to the rotating nuclei.

The following is a brief summary of the present work. The equation of motion of a perfectly elastic nuclear medium in the rotating frame of reference is derived. The effective forces, generated by the transformation of the equation of motion in the inertial system into that in the system of rotating coordinates, consists mainly of Coriolis and centrifugal forces. The case where the intial pressure is related to the external potential is also discussed. We have shown how the equation of motion of rotating nuclei was solved with the help of the perturbation method. The final result for the frequency of vi- bration of rotating nuclei is quite simple and its numerical evaluation becomes elementary. The angular velocity of nuclear rotation is briefly analyzed in relation to high angular momenta, which can be accomodated in nuclei. It is illustrated that for the $A=150$ region the Coriolis force dominates for small values $(\lesssim 0.05)$ of the ratio of angular velocity to resonance frequency, but the centrifugal force plays a prominent part in the shift of the split resonance energies for larger values of the ratio. It is seen that the rotational effect removes completely the azimuthal degeneracy of the giant resonance energies.

\section{ACKNOWLEDGMENTS}

We are grateful to Dr. P. Ring for fruitful discussions. Institut de Physique Nucléaire is an associated laboratory of CNRS.

\section{APPENDIX}

The radial integrals $I_{\mathrm{n}}$ and $I_{\mathrm{d}}$ of Eqs. (3.15) for constant density can be evaluated analytically using the explicit forms of the functions $U_{l}$ and $V_{l}$ of Eqs. (3.9) and (3.10). Thus,

$$
\begin{aligned}
\frac{I_{\mathrm{n}}}{\rho R_{0}^{5}}= & A_{l}^{2} \frac{1}{\xi^{4}} j_{l}^{2}(\xi)+2 A_{l} C_{l} \frac{1}{\xi^{2} \eta^{2}}\left\{\left[\xi \frac{d}{d \xi} j_{l}(\xi)+j_{l}(\xi)\right] j_{l}(\eta)+\frac{\xi^{2}}{\xi^{2}-\eta^{2}}\left[\xi j_{l+1}(\xi) j_{l}(\eta)-\eta j_{l}(\xi) j_{l+1}(\eta)\right]\right] \\
& +C_{l}^{2} \frac{1}{\eta^{4}}\left\{\left[\eta \frac{d}{d \eta} j_{l}(\eta)+j_{l}(\eta)\right] j_{l}(\eta)+l(l+1) j_{l}^{2}(\eta)+\frac{1}{2} \eta^{2}\left[j_{l}^{2}(\eta)-j_{l-1}(\eta) j_{l+1}(\eta)\right]\right\}, \\
\frac{I_{\mathrm{d}}}{\rho R_{0}^{5}}= & A_{l}^{2} \frac{1}{\xi^{4}}\left\{\xi j_{l}(\xi) \frac{d}{d \xi} j_{l}(\xi)+\frac{1}{2} \xi^{2}\left[j_{l}^{2}(\xi)-j_{l-l}(\xi) j_{l+1}(\xi)\right]\right\}+2 A_{l} C_{l} \frac{1}{\xi^{2} \eta^{2}} l(l+1) j_{l}(\xi) j_{l}(\eta) \\
& +C_{l}^{2} \frac{1}{\eta^{4}} l(l+1)\left\{j_{l}(\eta)\left[j_{l}(\eta)+\eta \frac{d}{d \eta} j_{l}(\eta)\right]+\frac{1}{2} \eta^{2}\left[j_{l}^{2}(\eta)-j_{l-1}(\eta) j_{l+1}(\eta)\right]\right\},
\end{aligned}
$$

where $\xi=h R_{0}$ and $\eta=k R_{0}, R_{0}$ being the radius of a spherical nucleus. $h$ and $k$ are defined in relation with Eqs. (3.9) and (3.10).

${ }^{1}$ S. Jang, Phys. Rev. C 33, 352 (1986).

${ }^{2}$ C. Y. Wong and N. Azziz, Phys. Rev. C 24, 2290 (1981); 25, 3110 (1982); C. Y. Wong, ibid. 25, 2787 (1982).

${ }^{3}$ G. F. Bertsch, Ann. Phys. (N.Y.) 86, 138 (1974); Nucl. Phys. A249, 253 (1975).

${ }^{4}$ J. O. Newton, B. Herskind, R. M. Diamond, E. L. Dines, J. E. Draper, K. H. Lindenberger, C. Schück, S. Smith, and F. S. Stephens, Phys. Rev. Lett. 46, 1383 (1981).

${ }^{5}$ N. Neergard, Phys. Lett. 110B, 7 (1982).

${ }^{6}$ A. V. Ignatyuk and I. N. Mikhailov, Yad. Fiz. 33, 919 (1981) [Sov. J. Nucl. Phys. 33, 483 (1982)].

${ }^{7}$ S. N. Fedotkin, I. N. Mikhailov, and R. G. Nazmitdinov, Phys. Lett. 121B, 15 (1983).

${ }^{8}$ P. Ring, L. M. Robledo, J. L. Egido, and M. Faber, Nucl. Phys. A419, 261 (1984).

${ }^{9}$ S. Jang and Z. Szymanski, Nucl. Phys. A436, 397 (1985).

${ }^{10}$ Z. Alterman, H. Jarosch, and C. L. Pekeris, Proc. R. Soc. London, Ser. A 252, 80 (1950); C. L. Pekeris, Z. Alterman, and H. Jarosch, Phys. Rev. 122, 1692 (1961); see also H.
Jeffreys, The Earth (Cambridge University, Cambridge, 1976).

${ }^{11}$ F. A. Dahlen, Geophys. J. R. Astron. Soc. 16, 329 (1968).

${ }^{12} \mathrm{~A}$. Bohr and B. R. Mottelson, Nuclear Structure (Benjamin, Reading, Mass., 1975), Vol. 2.

${ }^{13}$ Z. Szymanski, Fast Nuclear Rotation (Clarendon, Oxford, 1982), and references therein.

${ }^{14}$ S. Cohen, F. Plasil, and W. J. Swiatecki, Ann. Phys. (N.Y.) 82, 557 (1974).

${ }^{15}$ I. Ragnarsson, S. Aberg, H. B. Hakanson, and R. K. Sheline, Nucl. Phys. A361, 1 (1981).

${ }^{16} \mathrm{Z}$. Szymanski and I. Ragnarsson, in Proceedings of the XIVth Masurian Summer School on Nuclear Physics, Mikolajki, Poland, 1981, edited by B. Sikora and Z. Wilhelmi (Harwood, New York, 1984), p. 325.

${ }^{17}$ E. Balbutsev, Z. Vaishvila, and I. N. Mikhailov, Yad. Fiz. 35, 836 (1982) [Sov. J. Nucl. Phys. 35, 486 (1982)].

${ }^{18} \mathrm{P}$. Ring, paper presented at the Colloque GANIL, Giens, France, 1987 (unpublished); private communication. 